\title{
气动膜片式多材料微液滴按需喷射技术研究
}

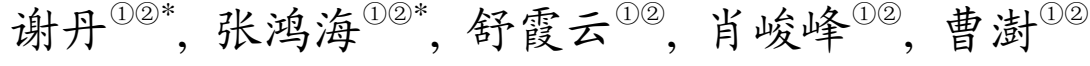 \\ (1) 华中科技大学机械科学与工程学院微系统中心, 武汉 430074; \\ (2) 武汉光电国家实验室微光机电系统研究部, 武汉 430074 \\ *E-mail: xiedan@ smail.hust.edu.cn; honghaizhang@tom.com \\ 收稿日期: 2009-09-24; 接受日期: 2009-12-21 \\ 国家自然科学基金(批准号: 50775087)资助项目
}

\begin{abstract}
摘要微滴喷射是通过产生微米级的液滴实现微量流体精确分配的技术之一，是一种不同 于传统减法制造的非接触式、数据驱动的加法式制造技术，代表了现代制造工艺新的发展方向. 本文提出了可用于多种材料的气动膜片式按需喷射技术并构建了用于在线观测并分析液滴形 成过程的基于延时触发的图像在线采集系统. 利用该装置, 进行了微液滴喷射的一致性分析, 研究了设备结构参数及控制参数与液滴大小及喷射速度的关系, 并分析了粘度及表面张力对 流体喷射过程的影响. 此外, 制作了直径约为 $160.5 \mu \mathrm{m}$ 的 $\mathrm{Sn}_{63} \mathrm{~Pb}_{37}$ 焊球和与基底接触面直径约 为 $346.94 \mu \mathrm{m}$ 的光固化胶胶滴阵列图型. 实验表明, 该气动膜片式按需喷射系统结构简单、可 靠性好、耐受高温, 可用于包括聚合物、低熔点胶材、高熔点金属的多材料微液滴喷射, 在微 电子和微系统封装、三维打印、有机半导体器件制作以及生命科学与化学分析等制造领域具有 较大的发展潜力.
\end{abstract}

关键词

微液滴 按需喷射 气动膜片式 多材料
微滴喷射是一种通过产生微米级的液滴实现微 量流体精确分配的技术之一, 是一种非接触式的直 写式制作技术 ${ }^{[1 ~ 5]}$. 与传统的减法工艺，如以钻、铣、 车为代表的机械加工工艺和以光刻、腐蚀为代表的半 导体工艺不同，微滴喷射是一种增材式制造技术，它 不仅减少工艺步骤、提高生产效率，还能节约材料、 具有极好的环境兼容性，此外，该技术与计算机控制 紧密结合, 可直接打印制造复杂的三维结构, 代表了 现代制造工艺新的发展方向. 微滴喷射技术从最初 的喷墨打印技术发展至今, 在应用于多种材料的喷 射制作中展现出了巨大的潜力, 如电子封装材料、生 物活性材料、聚合物材料、低熔点胶体材料、高熔点 金属材料等 ${ }^{[6 \sim 13]}$. 因此, 作为一种新兴的精密加工技
术，微滴喷射技术必将在微电子和微系统封装、三维 打印、有机半导体器件制作以及生命科学与化学分析 等领域发挥更大的作用.

微滴喷射技术主要有连续喷射式及按需式喷射 式 2 种模式 ${ }^{[1 ~ 5]}$. 连续式喷射的液滴以恒定的频率不 断的射出并被充电, 然后利用一电场对带电液滴进 行偏转控制, 使所需的液滴在指定位置沉积, 而剩下 的液滴则通过回收装置进行再利用, 连续式喷射由 于可控性差、精确定位难度大等缺点, 其使用范围远 不如按需喷射技术. 按需喷射是一种可控成滴技术, 它能按时按量将微液滴喷射沉积到指定位置. 其设 备主要有热气泡式、压电式以及气压直接驱动式. 热 气泡式是将喷头内的电热元件瞬时加热使与之接触 
的液体迅速汽化形成气泡, 气泡膨胀后将液体从喷 嘴中挤出形成液滴, 这种方法只适合容易产生气泡 的特制溶液的喷射 ${ }^{[14]}$. 压电式根据压电晶体的形状 及结构设计的差异有许多不同的形式, 其原理均是 在压电晶体上施加电压使其形状产生变化, 从而将 液体挤出形成液滴, 然而由于压电晶体的工作温度 不能高于其居里温度 $\left(350^{\circ} \mathrm{C}\right)$ 的一半, 因此当压电晶 体应用于熔点较高的物质的喷射时需设计非常复杂 的隔热结构用于保护压电晶体 ${ }^{[15,16]}$. 多伦多大学的 气压直接驱动式喷射装置是将压缩气体脉冲直接作 用在液面上形成微滴 ${ }^{[17 ~ 19]}$. 该装置结构简单、能工作 在高温条件下、可喷射材料范围广泛, 但驱动脉冲压 力直接作用在液面上, 液体腔液面会随着喷射的进 行逐渐下降, 因此驱动压力作用点的变化会导致喷 射不稳定并造成液滴的可控性较差. 因此, 本文提出 了气动膜片式多材料微液滴按需喷射技术, 以膜片 分离气体驱动腔及液体工作腔, 以压缩气体脉冲激 励膜片变形改变液体腔体积, 从而形成可控微液滴.

\section{1 气动膜片式按需喷射系统原理}

\section{1 气动膜片式按需喷射装置结构与工作原理}

按需式液滴喷射过程中, 射流液体会在腔体内 负压形成时产生面朝喷嘴方向速度, 这会导致射流 不稳定, 当负压足够大时, 射流将出现颈缩现象, 进 而断裂形成液滴. 不同的按需式喷射装置实现负压 的方式不尽相同, 热气泡式喷射装置是通过加热在 液体腔内产生气泡从而改变腔体气压; 而压电式装 置则通过压电晶体在驱动电路作用下的体积变形实 现驱动腔体的体积变化, 从而实现腔体内的压力变化.

气动膜片式微液滴按需喷射以压缩气体驱动膜 片变形改变液体腔体积, 从而导致液体从喷嘴中 射出形成液滴. 其结构如图 1 所示, 主要包括储料 腔、气体驱动腔、液体腔、节流阀、膜片、喷嘴以及

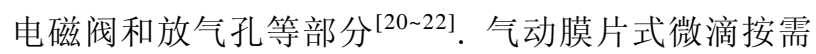
喷射过程如图 2 所示: 首先, 在储料腔上方施加背压 设定喷射初始状态, 此时, 由于表面张力的作用, 液 体将在喷嘴出口处形成稳定的“新月型”液面; 其次, 发出脉冲信号开启电磁阀, 压缩气体经由电磁阀进 入气体驱动腔, 腔内压力随之上升, 膜片随之变形向 下运动, 导致液体腔体积减小, 促使一部分液体挤出 喷嘴形成射流; 接着, 电磁阀关闭, 气体驱动腔内压

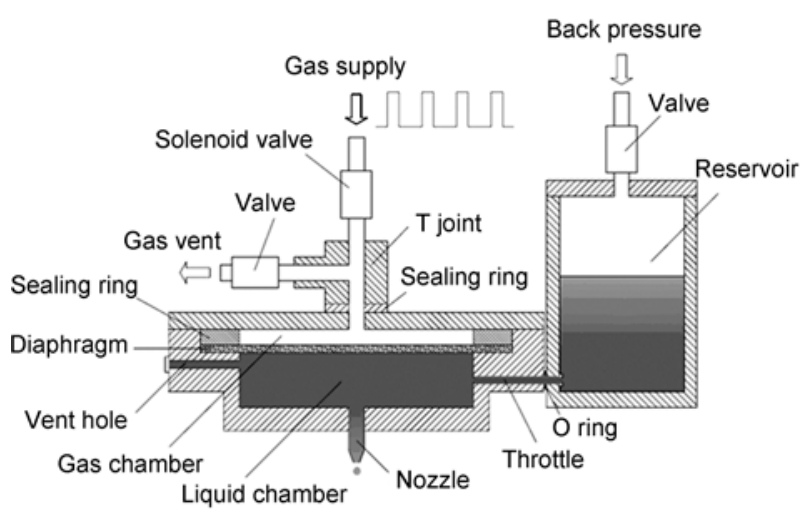

图 1 气动膜片式微滴喷射装置结构图

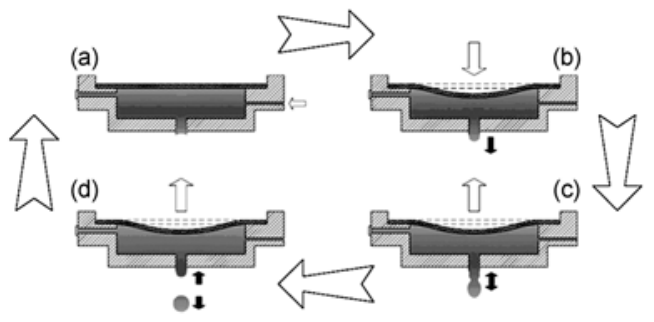

图 2 气动膜片式微滴喷射过程示意图

力通过出气孔释放, 导致液体腔内压力下降, 所形成 的射流靠近喷嘴处的部分速度减小甚至在负压作用 下的速度反向朝上，而射流前端由于惯性力的作用 速度依然朝下, 因此, 射流在两部分相反的作用力下 开始形成颈缩; 最后, 在表面张力、惯性力以及射流 后端液体的回拉作用下，颈部断裂形成液滴，而射流 后端部分将回缩至液体腔, 膜片也将回到初始状态, 开始下一轮的喷射.

\section{2 模型建立与分析}

图 3 所示为气动膜片式微滴喷射装置液体腔处 于工作状态时的物理简化模型, 膜片在气体驱动腔 内压力 $P_{C}$ 与液体腔内压力 $P_{F}$ 的共同作用下向下方变

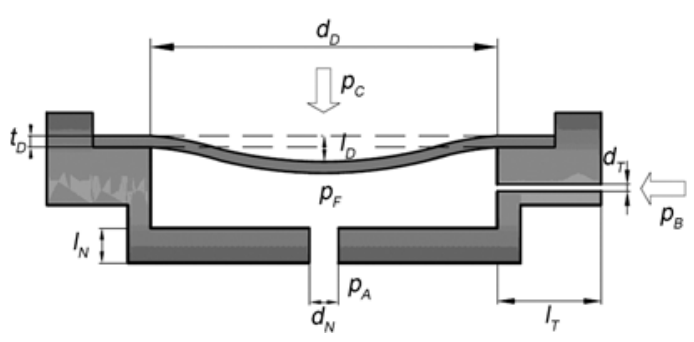

图 3 液体腔物理模型及参数 
形, 根据胡克定律, 忽略膜片动态过程中的惯性影响, 面积为 $S_{D}$ 的膜片中心位移 $l_{D}$ 与其两面压力差的方程 如下

$$
k_{D} l_{D}=\left(P_{C}-P_{F}\right) S_{D},
$$

$k_{D}$ 为膜片的抗弯刚度, 与膜片材料及形状有关

$$
k_{D}=\frac{E}{\left(1-v^{2}\right)} \frac{16 t_{D}^{3}}{d_{D}^{2}},
$$

常数 $E$ 和 $v$ 分别为膜片材料的弹性模量和泊松比, $t_{D}$ 和 $d_{D}$ 分别表示膜片的厚度和直径. 膜片变形所引起 的液体腔体积变化为

$$
V_{D}=\frac{1}{3} S_{D} l_{D}=\frac{\pi^{2}}{768} \frac{\left(1-v^{2}\right) d_{D}^{6}}{E t_{D}^{3}}\left(P_{C}-P_{F}\right) .
$$

设电磁阀开启的高电平时间为 $T$, 并假设所喷射 的液体是不可压缩流体, 液体腔、喷嘴及节流孔中的 液体运动为稳定流动状态, 可将液体腔、喷嘴及节流 孔中的流动视为层流. 因此, 由膜片所产生的平均体 积流率为

$$
Q_{D}=\frac{V_{D}}{T}=\frac{\pi^{2}}{768} \frac{\left(1-v^{2}\right) d_{D}^{6}}{E t_{D}^{3}} \frac{\left(p_{C}-p_{F}\right)}{T} .
$$

假定喷嘴流道为圆柱型, 根据哈根-泊肃叶方程, $\Delta p=\frac{32 \mu l U}{d^{2}},(\mu, U$ 表示液体粘度及流速), 可将其 体积流率表示为

$$
Q_{N}=U S_{N}=\frac{\pi d_{N}^{4}}{128 \mu l_{N}} \Delta p=\frac{\pi d_{N}^{4}}{128 \mu l_{N}}\left(p_{F}-p_{A}\right) .
$$

同理可得，节流口的体积流率为

$$
Q_{T}=U S_{T}=\frac{\pi d_{T}^{4}}{128 \mu l_{T}} \Delta p=\frac{\pi d_{T}^{4}}{128 \mu l_{T}}\left(p_{F}-p_{B}\right) .
$$

为简化计算, 在电磁阀开启膜片向下运动的过 程中, 即流体向喷嘴外形成射流的过程中忽略喷嘴 与节流口之间的相互作用, 将流入液体腔的体积流 率设为负, 而流出液体腔的体积流率设为正, 根据质 量守恒定理, 可得

$$
Q_{D}=Q_{T}+Q_{N} .
$$

在此气动膜片式按需喷射装置中, 可假设背压 值与喷嘴周边压力值与大气压相等, 即 $P_{B}=P_{A}=$ $P(1 \mathrm{~atm})$, 因此, 在脉冲上升沿作用过程中, 即膜片
向下变形至最大位置时, 由喷嘴中射出的液体体积 为

$$
V_{N}=Q_{N} T=\left(Q_{D}-Q_{T}\right) T .
$$

由方程(4), (5), (6), (7)和(8)可得

$$
V_{N}=\frac{p_{C}-p}{\frac{128 \mu l_{N}}{\pi d_{N}^{4} T}+\frac{768 E t_{D}^{3}}{\pi^{2}\left(1-v^{2}\right) d_{D}^{6}}\left(\frac{d_{T}^{4} l_{N}}{d_{N}^{4} l_{T}}+1\right)} .
$$

由公式(9)可以得出, 在膜片材料及流体确定的 条件下, 设备的结构参数及控制参数将对喷射形成 产生影响. (i)结构参数. 液滴直径主要与膜片直径、 喷嘴直径及节流口直径及膜片厚度相关. 膜片及喷 嘴直径越大, 液滴越大; 而节流口直径及膜片厚度越 大, 则液滴越小. 如果节流口过大, 会发生液体腔变 形时液体直接从节流口逆行返回至储料腔的现象, 在此情形下, 微液滴不会生成. (ii)控制参数. 脉冲高 电平时间及驱动压力的增大会导致液滴直径的增加. 背压的作用是为喷射设置一个初始状态和平衡位置, 通常背压值等于大气压时, 喷射即可正常进行, 然而 对于一些表面张力值特别小的液体, 必须将背压值 设为负压 (相对于大气压)才能稳定喷射, 否则, 液体 将直接从玻璃喷嘴中自动溢出, 无法形成稳定的喷 射初始状态.

\section{2 气动膜片式按需喷射实验系统构建}

气动膜片式按需喷射实验系统包括气压控制子 系统、电磁阀控制子系统、温度控制子系统、三维运 动平台控制子系统以及图像采集子系统, 如图 4 所示. 气压控制子系统用来对喷射系统提供背压和驱动压 力. 背压是一个静态压力, 只需加装减压阀即可. 而 驱动压力是一个脉冲压力, 需通过运动控制卡发出 脉冲信号经驱动电路控制电磁阀的快速开闭来实现. 我们采用加速电路驱动两位三通高速电磁阀可显著 降低了其标称开启时间, 这对于减小液滴的尺寸非 常有利, 下面将进行详细讨论. 温度控制子系统的作 用是将常温下无法喷射的物质加热将其粘度降至可 稳定喷射的范围, 通常为 1 30 cps. 本系统采用温控 仪、交流继电器及加热棒及加热圈组合的方式来实 现温度控制. 三维运动平台控制子系统用于精确控 制喷射液滴在基底上成型位置, 定位精度为 $\pm 0.1 \mu \mathrm{m}$. 


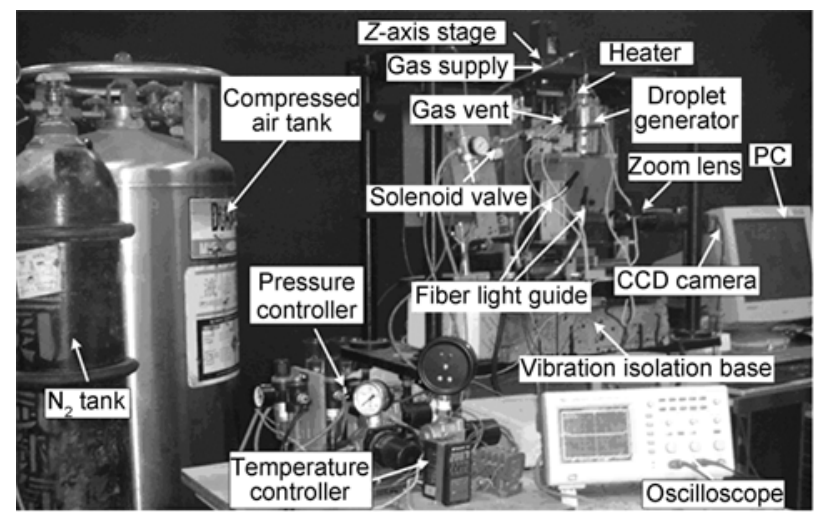

图 4 气动膜片式按需喷射实验系统

为观测并分析液滴形成过程, 本实验室搭建了基于 延时触发的图像采集子系统. 采用 CMOS 黑白数字 相机, 利用外部延时信号触发模式控制相机实现微 液滴喷射过程的图像采集, 其原理如图 5 所示, 控制 卡发出的脉冲一端经驱动加速电路激励电磁阀开启 形成喷射, 另一端经延时电路传送至触发端控制数 字相机的曝光成像, 通过调整延时电路的延时时间 可拍摄喷射后不同时刻的射流或液滴运动状态. 例 如, 本实验中, 采用 $50 \mu \mathrm{s}$ 的步距调整延时, 在喷射 开始后 $4 \mathrm{~ms}$ 的延时拍摄过程中, 可获得 80 张照片, 能基本反映出微液滴成型的过程, 并可从中提取微 液滴的尺寸及速度信息. 由于这种方法所拍摄到的 过程不是同一液滴的形成过程, 而是由不同液滴反 映的相同时刻液滴状态的组合图片, 因此, 喷射状态 的一致性及稳定性是采用基于延时触发的图像采集 子系统的至关重要的前提, 一致性测试实验及结果 将在 3.1 中详述.

微喷嘴是微滴喷射技术中最为关键的部件之一, 其直径大小、流道形状及内壁光滑程度都是影响喷射 能力的重要因素 ${ }^{[2]}$. 玻璃喷嘴具有出口直径及流道形

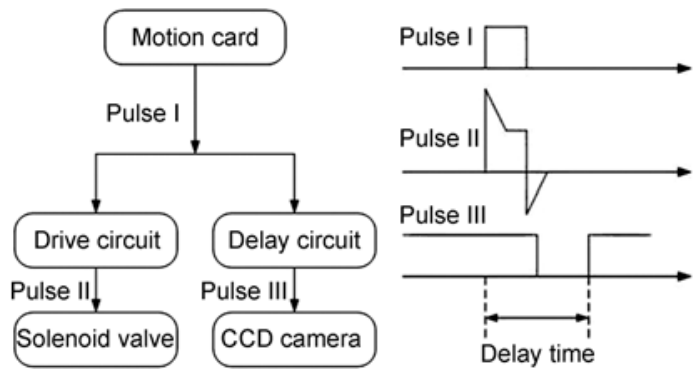

图 5 脉冲发生信号原理图
状可控、内壁光滑、成本低廉、可工作在 $400^{\circ} \mathrm{C}$ 高温 下、不易与喷射液体反映等特点. 图 6 为所制作的玻 璃喷嘴实物图, 其出口直径约为 $138.40 \mu \mathrm{m}$, 详细制 作方法另见他文.

\section{3 实验结果及分析}

根据以上的分析, 利用所搭建的气动膜片式按 需喷射实验系统对喷射的一致性、结构参数及控制参 数对液滴的大小及速度的影响进行了分析, 并进行 了应用试验研究, 结果分析如下.

\section{1 一致性分析}

按需喷射技术的特点是微液滴按时按量喷射成 型并在指定位置沉积成型, 这需要喷射过程具有良 好的可控性、高度的一致性和稳定性, 同时这也是延 时触发图像采集技术的前提条件. 本文采用 2 个指标 分析喷射的一致性, 喷嘴出口距离液滴中心的距离 $L_{d}$ 以及微液滴的直径 $D_{d}$, 如图 7 所示. 文中研究了
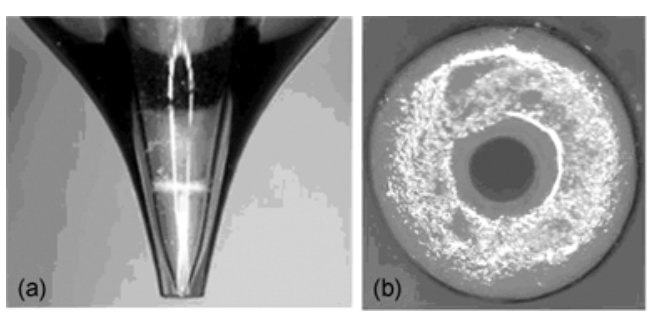

图 6 玻璃喷嘴

(a)外观图( $\times 50)$; (b)喷嘴出口 $(\times 200)$

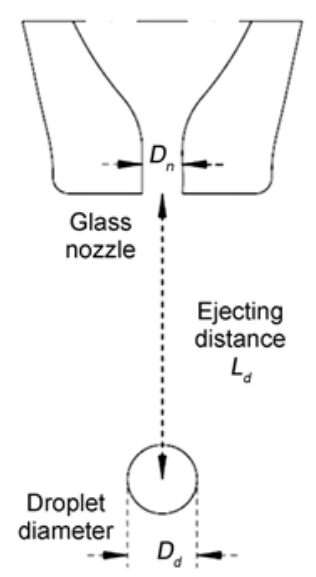

图 7 液滴喷射过程参数定义 
5 和 $50 \mathrm{~Hz}$ 时喷射发生后不同时间间隔水液滴的 $L_{d}$ 和 $D_{d}$ 值及其变化率. 工作参数为: 驱动压力 0.075 $\mathrm{MPa}$, 脉宽 $1.8 \mathrm{~ms}$, 喷嘴直径 $65.78 \mu \mathrm{m}$, 膜片直径 $20 \mathrm{~mm}$. 由图 8 可知, $L_{d}$ 随着时间间隔的延长逐渐增 大, 其波动范围也相应加大, 因此, 当采用液滴直接 沉积成型的方法进行图形制作时, 基底距离喷嘴出 口处越近越好, 通常不超过 $2 \mathrm{~mm}$. 而微液滴形成后 其直径具有良好的均匀性, 变化小于 $1 \%$.

\section{2 膜片大小对喷射的影响}

本实验以纯水为研究对象, 实验条件为频率 2 $\mathrm{Hz}$, 脉冲宽度 $1.8 \mathrm{~ms}$, 喷嘴直径 $65.78 \mu \mathrm{m}$, 液体腔可 更换 4 种尺寸的膜片, 其直径分别为 14, 20, 30 和 $38 \mathrm{~mm}$, 对于不同大小的膜片, 需调整所施加的驱动 压力得到稳定的喷射状态, 随膜片尺寸的增大, 所需 驱动压力减小, 分别为 $0.1,0.075,0.065$ 和 $0.06 \mathrm{MPa}$. 图 9 中实验结果表明, 4 种膜片直径所对应的液滴直 径以及液滴速度分别为 $141.79,152.61,156.72$ 和 $160.45 \mu \mathrm{m}$ 以及 $0.95,0.81,0.78$ 和 $0.59 \mathrm{~m} / \mathrm{s}$. 可见, 随 着膜片直径的增大, 液滴直径随之增大, 这与公式 (9)中的结论吻合, 而喷射速度随之降低与喷射压力 的调整有关.

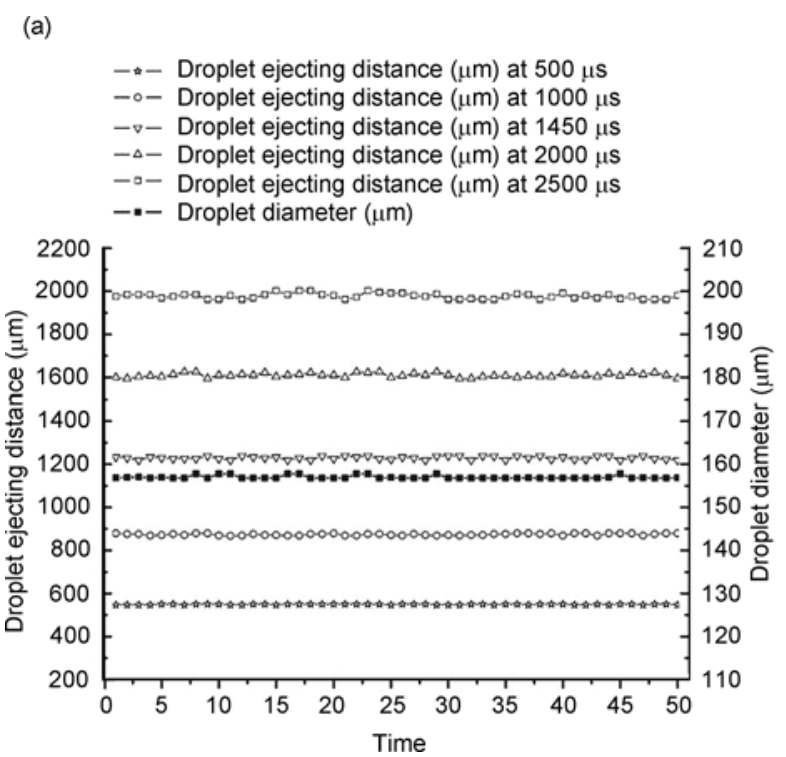

\section{3 控制参数对喷射的影响}

\subsection{1 驱动压力}

本实验以纯水为研究对象, 实验条件为频率 5 $\mathrm{Hz}$, 脉冲宽度 $1.8 \mathrm{~ms}$, 喷嘴直径 $72.11 \mu \mathrm{m}$, 膜片直径 为 $20 \mathrm{~mm}$, 驱动压力在 $0.065 \sim 0.075 \mathrm{MPa}$ 的范围喷射 处于稳定的状态, 当压力过小时, 喷嘴出口处的液面 会随着脉冲压力的激励上下抖动，但无法克服表面 张力形成液滴, 而压力过大时则会产生卫星滴, 影响 喷射的稳定性. 如图 10 所示, 4 个压力所对应的液滴 直径和喷射速度分别为 $138.06,141.59,146.33$ 和 $149.25 \mu \mathrm{m}$ 以及 $0.53,0.67,0.77$ 和 $0.90 \mathrm{~m} / \mathrm{s}$. 随着驱 动压力的增大, 液滴直径和喷射速度几乎呈线性增 长. 这与公式(9)中的驱动压力增大导致液滴直径增 大的结论相符.

\subsection{2 操作频率}

本实验以纯水为研究对象, 实验条件为驱动压 力 $0.075 \mathrm{MPa}$, 脉冲宽度 $1.8 \mathrm{~ms}$, 喷嘴直径 $65.78 \mu \mathrm{m}$, 膜片直径为 $20 \mathrm{~mm}$, 操作频率分别为 $2,4,5,8,10,20$, $40,50,80$ 和 $100 \mathrm{~Hz}$, 实验中发现, 当喷射稳定进行 时, 只调节频率而其他参数不变时, 喷射依然处于稳 定的状态. 如图 11 所示, 随着频率的增加, 喷射速度 降缓, 其值由 $0.98 \mathrm{~m} / \mathrm{s}$ 降至 $0.25 \mathrm{~m} / \mathrm{s}$, 图 8 中也可得

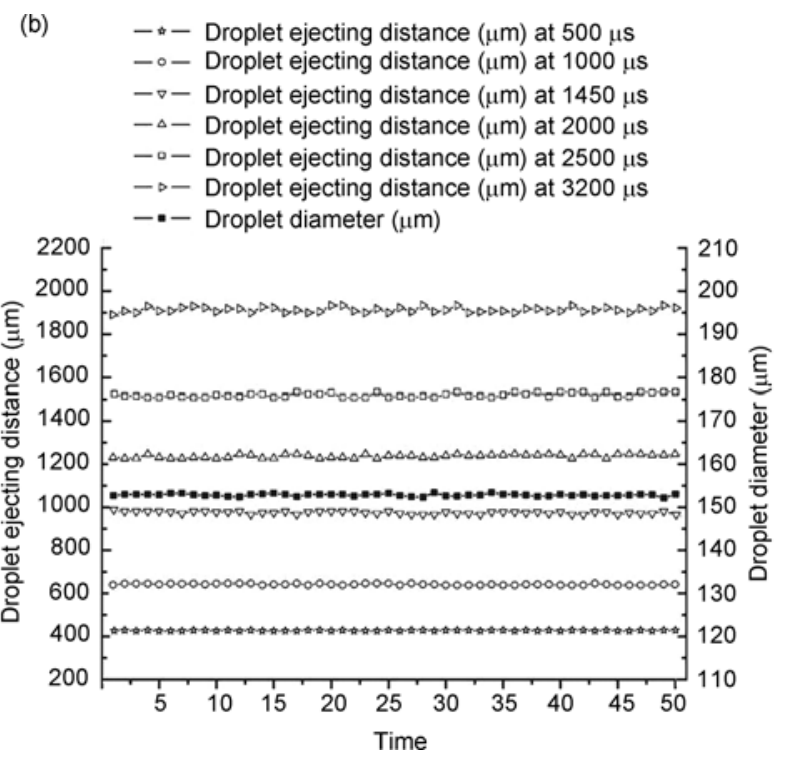

图 8 喷射过程的一致性实验

(a) $5 \mathrm{~Hz}$; (b) $50 \mathrm{~Hz}$ 


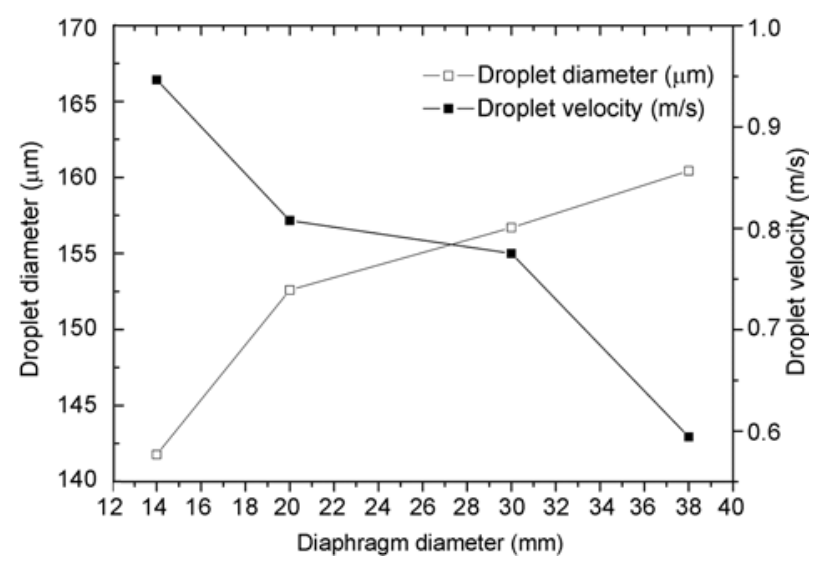

图 9 膜片直径与微液滴直径及速度的关系

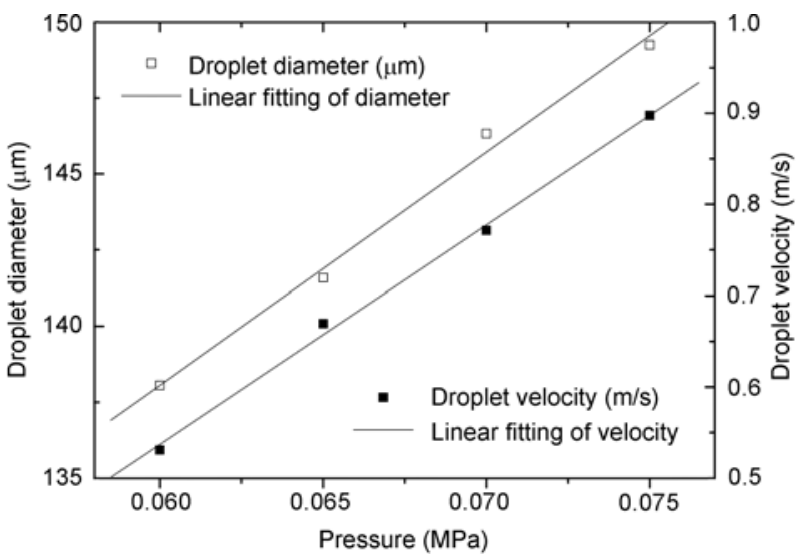

图 10 驱动压力与微液滴直径及速度的关系

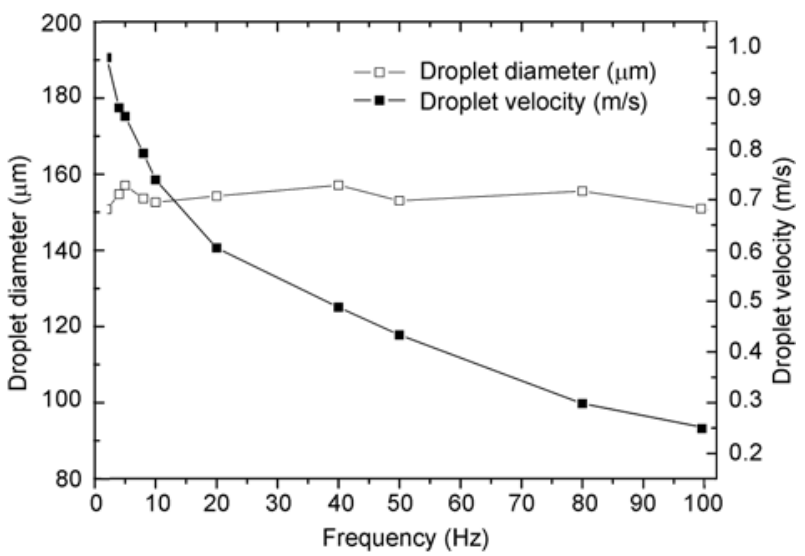

图 11 操作频率与微液滴直径及速度的关系

出频率增加导致速度减慢的结论, 然而, 频率的变化 对液滴直径的变化几乎没有影响, 其值在 150.75 至 $157.09 \mu \mathrm{m}$ 的区间内无规律变化.

\subsection{3 脉冲宽度}

本实验以纯水为研究对象, 实验条件为驱动压 力 $0.06 \mathrm{MPa}$, 操作频率 $2 \mathrm{~Hz}$, 喷嘴直径 $65.78 \mu \mathrm{m}$, 膜 片直径为 $20 \mathrm{~mm}$, 脉冲宽度分别为 $1.5,1.6,1.7,1.8$, $1.9,2.0$ 和 $3.0 \mathrm{~ms}$, 从图 12 中可以看到, 脉宽的调节 对液滴的大小及速度影响较大, 其值分别由 123.13 增至 $152.99 \mu \mathrm{m}$ 以及 0.24 增至 $0.98 \mathrm{~m} / \mathrm{s}$, 尤其在 1.5 2.0 ms 的范围内, 直径与速度变化十分显著. 此 结论与公式(9)中的脉冲宽度增大导致液滴直径增大 的结论相符.

\section{4 多材料按需喷射实验}

\subsection{1 甘油/水溶液按需喷射实验}

气动膜片式按需喷射装置的目标是能够实现多 种材料, 包括低熔点金属、光学及电子材料、生物活 性溶液. 这些材料包括牛顿流体及非牛顿流体, 性质 迥异，因此，描述不同性质流体的单个液滴的喷射形 成过程十分重要. 根据文献 [23，24]可知，可形成稳 定喷射的流体其粘度为 $0.5 \sim 30 \mathrm{cps}$, 表面张力为 20 70 dyn $/ \mathrm{cm}$, 对于某些高粘度液体, 可通过加温的 方式降低粘度进行喷射. 本节中, 采用 4 种溶液进行 了按需喷射的实验, 纯水、水/甘油溶液(质量比: 48/52), 水/甘油/异丙醇溶液 (质量比: 34/53/13), 水/ 甘油溶液(质量比: 60/40), 其物理性质见表 1 .

使用频率 $4 \mathrm{~Hz}$ 、脉宽为 $1.5 \mathrm{~ms}$ 的脉冲信号驱 动 4 种溶液, 稳定喷射的驱动压力分别为 $0.075,0.2$, 0.16 和 $0.48 \mathrm{MPa}$, 所使用的膜片直径为 $20 \mathrm{~mm}$, 喷 嘴直径为 $138.40 \mu \mathrm{m}$. 图 13 展示的过程图片清晰地

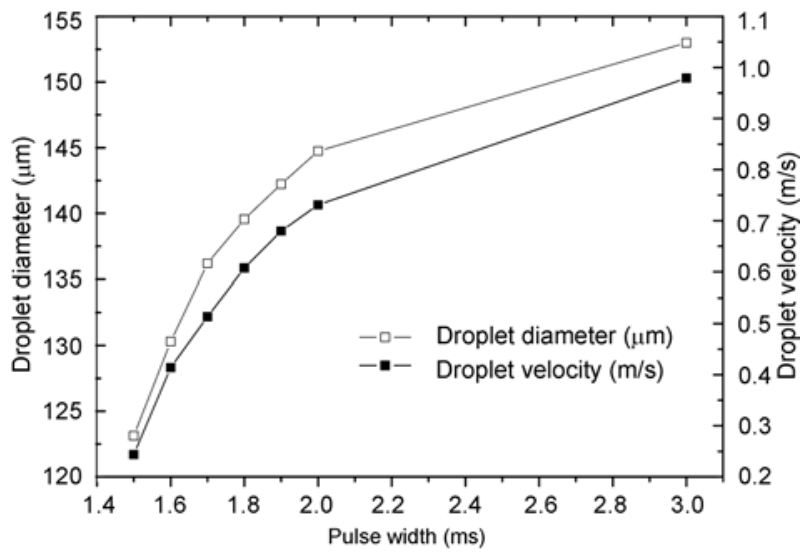

图 12 脉冲宽度与微液滴直径及速度的关系 


\section{表 1 溶液性质}

\begin{tabular}{cccc}
\hline Fluids (mass ratio) & $\begin{array}{c}\text { Density } \\
\left(\mathrm{g} / \mathrm{cm}^{3}\right)\end{array}$ & $\begin{array}{c}\text { Viscosity } \\
(\mathrm{mPa} \cdot \mathrm{s})\end{array}$ & $\begin{array}{c}\text { Surface tension } \\
(\mathrm{mN} / \mathrm{m})\end{array}$ \\
\hline $\begin{array}{c}\text { Water } \\
\text { Glycerin/Water } \\
(48 / 52)\end{array}$ & 1.00 & 1.0 & 73 \\
$\begin{array}{c}\text { Glyerin/Water/Isopro } \\
\text { panol (34/53/13) } \\
\begin{array}{c}\text { Glycerin/Water } \\
(60 / 40)\end{array}\end{array}$ & 1.12 & 5.0 & 70 \\
\hline
\end{tabular}

(a)

(1)

$1100 \mu \mathrm{s} 1300 \mu \mathrm{S} 1500 \mu \mathrm{s} 1800 \mu \mathrm{s} 2000 \mu \mathrm{s} 2200 \mu \mathrm{s} 2400 \mu \mathrm{s} 3500 \mu \mathrm{s}$

(b)

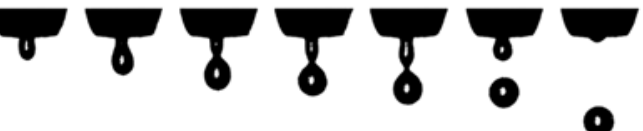

$1200 \mu \mathrm{s} 1400 \mu \mathrm{s} 1800 \mu \mathrm{s} 2300 \mu \mathrm{s} 2500 \mu \mathrm{s} 2800 \mu \mathrm{s} 2900 \mu \mathrm{s} 4000 \mu \mathrm{s}$ (c)

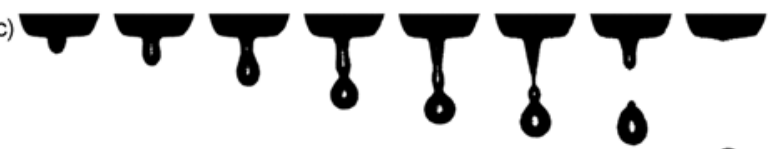

-

$1200 \mu \mathrm{S} 1400 \mu \mathrm{S} 1800 \mu \mathrm{s} 2300 \mu \mathrm{s} 2700 \mu \mathrm{s} 2900 \mu \mathrm{S} 3100 \mu \mathrm{s} 4000 \mu \mathrm{S}$

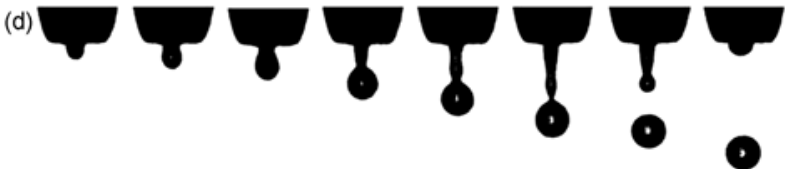

$1200 \mu \mathrm{S} 1400 \mu \mathrm{s} 1700 \mu \mathrm{s} 2200 \mu \mathrm{s} 2600 \mu \mathrm{s} 3100 \mu \mathrm{s} 3300 \mu \mathrm{s} 4000 \mu \mathrm{s}$

图 13 喷射过程图片

描述了喷射成滴的过程: 喷射、伸长、颈缩、断裂、 振荡、成滴. 可见, 4 种溶液拉断的时间分别为 2400 , 2800,2900 和 $3100 \mu \mathrm{s}$, 因此, 流体粘度越大, 所需的 驱动力越大, 而将所形成射流拉断所需的时间越长. 如图 13(a), (b)和 (d)可看出, 随着粘度的增大, 射流 伸长的长度越大, 而所形成的液滴也会越大. 表面张 力的作用对所需驱动压力影响较小, 然而由于表面 张力影响液滴的成型能力, 因此, 较小的表面张力会 造成流体射出伸长段的剧烈振荡, 如图 13(c) 所示. 因此, 表面张力所引起的剧烈振荡会使伸长段断裂 形成一个或多个卫星滴, 引起喷射的不稳定, 这是需 避免的现象. 表 2 所示为 4 种液滴的大小及速度.

\subsection{2 金属焊料按需喷射实验}

选用 $\mathrm{Sn}_{63} \mathrm{~Pb}_{37}$ 焊料进行按需喷射实验. 其熔点为
表 24 种液滴大小及速度

\begin{tabular}{cccc}
\hline $\begin{array}{c}\text { Liquids } \\
(\text { mass ratio })\end{array}$ & $\begin{array}{c}\text { Diameter } \\
(\mu \mathrm{m})\end{array}$ & $\begin{array}{c}\text { Volume } \\
(\mathrm{nL})\end{array}$ & $\begin{array}{c}\text { Velocity } \\
(\mathrm{m} / \mathrm{s})\end{array}$ \\
\hline $\mathrm{W}(1)$ : DI water & 261.19 & 9.33 & 0.3651 \\
Glycerin/Water 48/52 & 298.51 & 13.93 & 0.2882 \\
$\begin{array}{c}\text { Glycerin/Water/Isopropanol } \\
\text { 134/53/13 }\end{array}$ & 291.04 & 12.91 & 0.2661 \\
Glycerin/Water, 60/40 & 335.82 & 19.83 & 0.3397 \\
\hline
\end{tabular}

$183^{\circ} \mathrm{C}, 300^{\circ} \mathrm{C}$ 时其各项物理参数如下, 粘度 $2 \sim 5 \mathrm{cps}$, 表面张力 470 480 dyn $/ \mathrm{cm}$, 密度约 $7.8 \times 10^{3} \mathrm{~kg} / \mathrm{m}^{3}$. 由 于焊料喷射在高温状态下进行, 需使用高温密封胶 及密封圈保证腔体的密封. 此外, 熔化焊料表面极易 氧化, 氧化层会影响射流的断裂成滴, 严重时甚至会 堵塞喷嘴, 为保证喷射正常进行, 需将喷嘴附近气体 氛围的氧浓度设置为低于 $35 \mathrm{ppm}$, 因此, 设计制作 了同轴供气装置, 在喷嘴上方附近缓慢供应氮气, 在 其周围形成局部低氧浓度氛围. 具体实验参数为: 喷 嘴直径 $100 \mu \mathrm{m}$, 膜片直径为 $20 \mathrm{~mm}$, 驱动压力为 0.25 $\mathrm{MPa}$ ，脉宽为 $1.8 \mathrm{~ms}$, 频率为 $5 \mathrm{~Hz}$, 工作温度 $250^{\circ} \mathrm{C}$. 图 14 所示为硅油中微焊球的照片, 平均直径为 160.5 $\mu \mathrm{m}$ ，尺寸偏差小于 $2 \%$.

\subsection{3 光固化胶按需喷射实验}

选用 Evervide 公司的光固化胶进行喷射实验, 其粘度约为 $21.5 \mathrm{cps}$, 硬化条件为 $2000 \mathrm{mj} / \mathrm{cm}^{2}$. 该实 验需在黄光条件下进行, 喷射胶滴沉积成型后, 使 用紫外灯进行固化. 具体实验参数为: 喷嘴直径 $72.11 \mu \mathrm{m}$, 膜片直径为 $38 \mathrm{~mm}$, 驱动压力为 $0.5 \mathrm{MPa}$, 脉宽为 $1.8 \mathrm{~ms}$. 图 15 所示为所形成光固化胶滴阵列 及其侧面的照片，与基底接触面的平均直径为 $346.94 \mu \mathrm{m}$, 尺寸偏差小于 $3 \%$.

\section{4 结论}

本文提出了一种气动膜片式微液滴按需喷射系

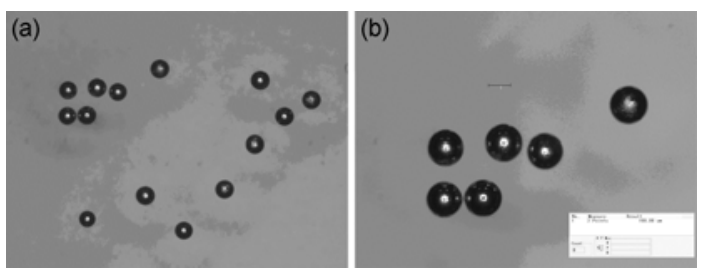

图 14 微焊球

(a) $\times 100$; (b) $\times 200$ 


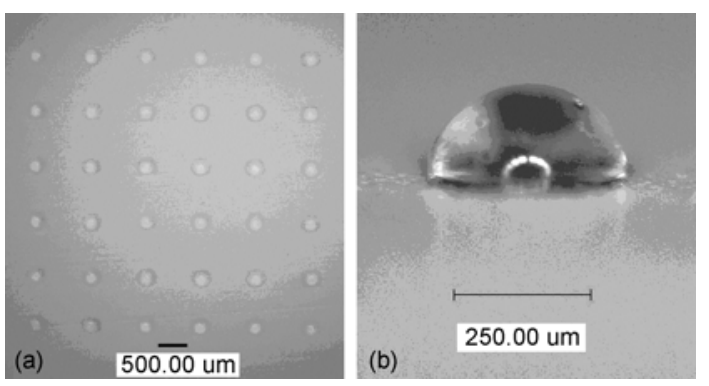

图 15 光固化胶胶滴图型

(a) $6 \times 6$ 阵列 $\times 20$; (b) 单个凸点侧视图 $\times 200$

统, 以膜片为驱动部件, 以压缩气体脉冲为驱动源, 通过膜片变形实现液体腔的体积变化从而产生液滴,
通过调节装置的结构参数如膜片大小、喷嘴大小及 控制参数如驱动压力、脉冲宽度及操作频率可控制 液滴大小及喷射速度. 此外, 基于延时触发的图像 在线采集系统可用于拍摄微液滴喷射的过程, 并可 获取液滴大小及速度等信息. 利用该设备制作了微 焊球及光固化胶胶滴阵列图型. 实验结果表明, 该 气动膜片式按需喷射系统结构简单、可靠性好、易 于操作及维修、耐受高温，可用于包括聚合物、低熔 点胶材及高熔点金属的多材料的微液滴喷射, 在微 电子和微系统封装、三维打印、有机半导体器件制 作以及生命科学与化学分析等制造领域具有极大的 发展潜力。

\section{参考文献}

1 Hon K K B, Li L, Hutchings I M. Direct writing technology-Advances and developments. CIRP Annals Manuf Tech, 2008, 57: 601-620

2 Lee E R. Microdrop Generation. Boca Raton: CRC Press, 2002

3 Burgold J, Weise F, Fischer M, et al. Evolution and operating experiences with different drop-on-demand systems. Macromol Rapid Commun, 2005, 26: 265-280

4 Gao C, Huang S X. Progress in inkjet technique and its applications. J Inorg Mater, 2004, 19: 714-722

5 Huang H, Qi L. Uniform droplets forming technology and application. Manuf Tech Mach Tool, 2008, 19: 59-63

6 Wallace D, Hayes D, Chen T, et al. Think additive: Ink-jet deposition of materials for MEMS packaging. In: 6th Topical Workshop on Packaging of MEMS and Related Micro-Nano-Bio Integrated Systems. California: Long Beach, 2004

7 Hayes D, Grove M, Cox W. Development and application by ink-jet printing of advanced packaging materials. In: International Symposium on Advanced Packaging Materials: Processes, Properties and Interfaces. Georgia: Braselton, 1999

8 Lee T M, Kang T G, Yang J S, et al. Drop-on-demand solder droplet jetting system for fabricating microstructure. IEEE Trans Electron Pack Manuf, 2008, 31: 202-210

9 Gans B J de, Duineveld P C, Schubert U S. Inkjet printing of polymers: State of the art and future developments. Adv Mater, 2004, 16: $203-213$

10 Mott M, Song J H, Evans J R G. Microengineering of ceramics by direct ink-jet printing. J Am Ceramic Society, 1999, 82: 1653-1658

11 Fan K C, Chen J Y, Wang C H, et al. Development of a drop-on demand droplet generator for one-drop-fill technology. Sens Actuators A: Phys, 2008, 147: 649-655

12 Duineveld P C, Kok M M de, Buechel M. Ink-jet printing of polymer light-emitting devices. In: Proceedings of SPIE, the International Society for Optical Engineering. California: San Diego, 2002

13 Zaugg F G, Wagner P. Drop-on-demand printing of protein biochip arrays. Mater Res Society, 2003, 28: 837-842

14 Cabal A, Ross D S, Lebens J A, et al. Thermal actuator with optimized heater for liquid drop ejectors. Sens Actuators A: Phys, 2005, 123: $531-539$

15 Brünahl J, Grishin A M. Piezoelectric shear mode drop-on-demand inkjet actuator. Sens Actuators A: Phys, 2002, 101: 371 - 382

16 Wang D A, Cheng C H, Hsieh Y H, et al. Analysis of an annular PZT actuator for a droplet ejector. Sens Actuators A: Phys, 2007, 137: $330-337$

17 Cheng S, Chandra S. A pneumatic droplet-on-demand generator. Experiments Fluids, 2003, 34: 755-762

18 Cheng S X, Li T, Chandra S. Producing molten metal droplets with a pneumatic droplet-on-demand generator. J Mater Proc Tech, 2005, 159: $295-302$

19 Chandra S, Rahim J. Apparatus and method for generating droplets. US patent, 6446878, 2002

20 Zhang H H, Shu X Y, Xie D, et al. A pneumatically actuator diaphragm-driven micro-droplet generator. Chinese patent 2009103055151 1, 2009

21 Shu X Y. Mechanism and Experimenal Study of Pneumatically Actuator Diaphragm-driven Micro-droplet Generator for Molten Metal. Dissertation of Doctoral Degree. Wuhan: Huazhong University of Science and Technoloty, 2009

22 Xiao J F. Study on Pneumatic Diaphragm-based Micro Droplet Generator system. Dissertation of Masteral Degree. Wuhan: Huazhong University of Science and Technoloty, 2009

23 Jang D, Kim D, Moon J. Influence of fluid physical properties on ink-jet printability. Langmuir, 2009, 25: 2629-2635

24 Fluid Properties Effects on Ink-jet Device Performance. Microfab Technote, 99-02 